\title{
Development of a highly sensitive spectral camera for cartilage monitoring using fluorescence spectroscopy
}

\author{
A. Kuehn, A. Graf, U. Wenzel, S. Princz, H. Mantz, and M. Hessling \\ Ulm University of Applied Sciences, Department of Mechatronics and Medical Engineering, \\ Albert-Einstein-Allee 55, 89081 Ulm, Germany \\ Correspondence to: M. Hessling (hessling@hs-ulm.de)
}

Received: 7 July 2015 - Revised: 2 September 2015 - Accepted: 4 September 2015 - Published: 16 September 2015

\begin{abstract}
The Ulm University Medical Center and the Ulm University of Applied Sciences are developing a bioreactor to grow facial cartilage using the methods of tissue engineering. To ensure a sufficient quality of the cartilage prior to implantation, the cartilage growth has to be monitored continuously. Current cartilage analysis methods are destructive so that analysed cartilage sample is no longer suitable for implantation. Alternatively, it seems feasible to analyse cartilage during the cultivation process and before implantation using fluorescence spectroscopy after UV light excitation. This approach is non-invasive and allows an evaluation of the cartilage in terms of composition and quality. Cultured cartilage implants can reach sizes of several square centimetres and therefore it is necessary to examine it over its entire area. For recording fluorescence spectra of different spots of the cartilage sample, a highly sensitive spectral camera is being developed in two steps. The first step is a one-dimensional spectral camera that is able to record fluorescence spectra along a sample line. The second step enables the detection of spectra over the required two-dimensional sample area. This approach is based on computed tomography imaging spectrometry (CTIS) and allows non-invasive distinguishing of the most important cartilage compounds collagen I and collagen II.
\end{abstract}

\section{Introduction}

The importance of cartilage for the function of joints is commonly accepted, but facial cartilage defects are also crucial. Missing or defect facial cartilage caused by diseases or accidents (e.g. of the ear or nose) can lead not only to serious psychological problems. In addition it may cause the loss of physiological functions and even lead to deadly peril if the trachea is affected. To restore the function and remedy disfigurement there are several possible treatment options. One common method is to substitute the defects with artificial implants made of silicone, metals and metal alloys or synthetics. Another option is replacing the lost tissue with an allograft. However, both methods involve the risk of body rejections and the demand for allografts can only be supplied to a very small extent because of a lack of immunologically compatible donors.

The most promising alternative is to create personalized implants from the patient's own cells using the methods of tissue engineering. Tissue engineering is a new interdisci- plinary field that applies the principles of engineering and life science to develop artificial biological substitutions to replace, restore or improve tissue function (Skalak and Fox, 1988). The cells are taken from the donor and multiplied in vitro. Subsequently, the cells are seeded onto a so-called scaffold, which is an empty three-dimensional framework of the desired shape that is colonized by the patient's cells (Langer and Vacanti, 1993). The important advantage of this autologous cartilage tissue is that the immune response is kept to a minimum. A major problem in tissue engineering is the possibility for cells to dedifferentiate during cultivation and lose or change their characteristic morphological and functional properties (Von der Mark et al., 1977; Lefebvre et al., 1990; Sittinger et al., 1996). In this case the tissue would not be suitable for implantation any longer.

To recognize and avoid morphological and functional changes, the cartilage growth has to be monitored continuously. Collagen type I and II are cartilage compounds with the highest concentration and are of large importance for the 


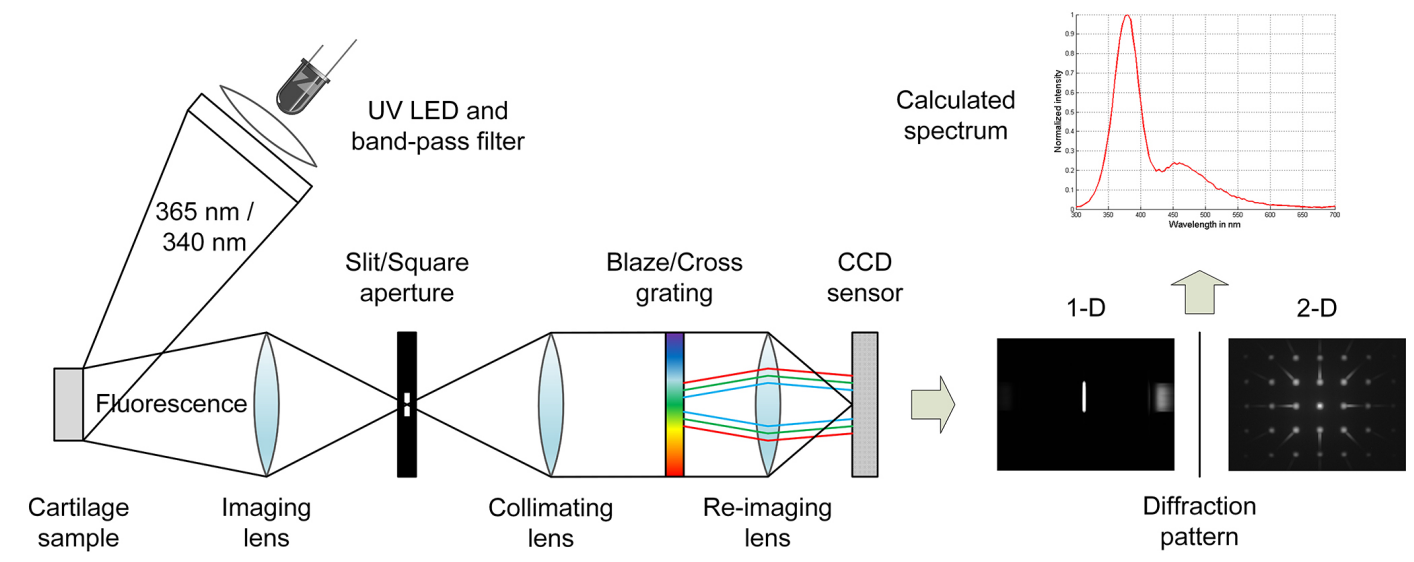

Figure 1. Simplified scheme of one-/two-dimensional spectral camera setup and spectrum generation.

mechanical properties. Unfortunately, the current standard method for cartilage analysis is the immune histochemical staining which destroys cartilage components and makes the cartilage tissue useless for implantation. An alternative, nondestructive approach is to determine important cartilage components like collagen I, collagen II, elastin and glycosaminoglycan using their fluorescence spectra. The different collagen types and elastin are among the most important fluorophores in the extracellular matrix (Tuan and Brian, 2003). The collagen fluorescence is caused by pyridinoline, a crosslinking compound of collagen (Uchiyama et al., 1981; Kirkpatrick, 2006). Because of differences in the fluorescence properties of the different collagen types, it is possible to distinguish collagen I and II non-invasively by recording the fluorescence spectra after UV excitation.

Because of the large size of future cartilage implants it would be insufficient to judge a potential implant with an area of several square centimetres by the properties determined at a single spot. The complete cartilage tissue area has to be examined. Therefore a spectral camera is needed that records sample spectra of the whole tissue. Unfortunately, the fluorescence radiation is very weak and the emission spectrum wavelength ranges between 380 and $500 \mathrm{~nm}$.

Presently, there is no commercial spectral camera with the necessary high sensitivity and the required spectral region. Therefore the development of a highly sensitive and even low-cost spectral camera has been started in two steps. The first step is a setup for recording fluorescence spectra along a single line on the sample. The second step is based on a modification of computed tomography imaging spectrometry (CTIS) as described in Habel et al. (2012), but several additional measures had to be taken to reach the desired sensitivity in the wavelength region $380-500 \mathrm{~nm}$ for recording spectra over a two-dimensional sample area.

This article is a more detailed and extended version of the proceedings paper of Kuehn et al. (2015) with additional material for the one-dimensional and the two-dimensional spectral camera.

\section{Setup and cartilage samples}

In both systems presented in this study (Fig. 1) the fluorescence excitation is performed with one of two UV LEDs: a $365 \mathrm{~nm}$ LED with an optical power of $410 \mathrm{~mW}$ at $700 \mathrm{~mA}$ (LED Engin, LZ1-00U600) or a $340 \mathrm{~nm}$ LED (Epigap Optronic, EOLD-340-TO) with a radiant flux of $1.4 \mathrm{~mW}$ at an electric current of $20 \mathrm{~mA}$. To avoid LED radiation in the spectral range of the fluorescence detection, the band-pass filters BP 365/12 (Delta Optical Thin Film A/S, LF101653), the band-pass filter with $340 \mathrm{~nm}$ central wavelength, and $26 \mathrm{~nm}$ band-width (Edmund Optics, Techspec, \#84-092) filter are mounted between the UV LED and sample. Using a homogenizing light pipe (Edmund Optics, Techspec, $10 \mathrm{~mm} \times 125 \mathrm{~mm}$, \# 63-097) - not depicted in Fig. 1 - even cartilage samples of several square centimetres are illuminated homogeneously.

\subsection{One-dimensional spectral camera}

The emitted fluorescence of the cartilage sample is focused on a $20 \mu \mathrm{m}$ width slit by a $35 \mathrm{~mm}$ lens (Edmund Optics, Techspec, \#59-872). Behind the slit, the light diverges and is collimated by a $25 \mathrm{~mm}$ lens (Edmund Optics, Techspec, \#59-871). The collimated light hits a blaze grating with 300 lines $\mathrm{mm}^{-1}$ (Edmund Optics, UV Transmission Grating Beamsplitter, $25 \mathrm{~mm} \times 25 \mathrm{~mm}$, \# 85-291) which diffracts the light. Subsequently, a $30 \mathrm{~mm}$ lens (Thorlabs, AC254-030-AML) reimages the light onto a CCD sensor of a monochromatic astronomy camera (Atik $314 \mathrm{~L}+$ ). The built-in CCD sensor (Sony ICX-285AL) has a resolution of $1392 \times 1040$ pixels with a pixel size of $6.45 \mu \mathrm{m} \times 6.45 \mu \mathrm{m}$. In order to reduce signal noise, a Peltier element cools the CCD sensor of the camera by $27^{\circ} \mathrm{C}$. The combination of this low noise but highly sensitive monochrome camera and fast lenses with numerical apertures of about 0.3 is judiciously selected to detect the expected weak fluorescence signals. Using image analysis of the recorded diffraction pattern (Fig. 1), the fluores- 

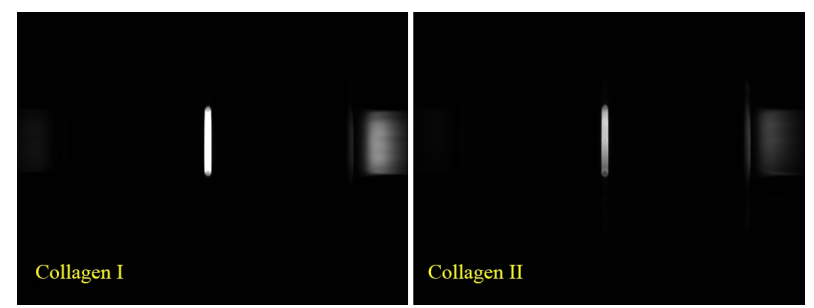

Figure 2. Diffraction spectra of collagen I and II fluorescence with $365 \mathrm{~nm}$ excitation. Integration time: $10 \mathrm{~s}$ for collagen I, $60 \mathrm{~s}$ for collagen II.

cence spectrum of every point of the line on the sample can be calculated by the grid in Eq. (1) that converts positions on the CCD chip into wavelengths.

$\lambda=\frac{g d_{m}}{m \sqrt{f^{2}+d_{m}^{2}}}$

with the grid constant $g$, the focal length of the third lens $f$ and the distance between zeroth and $m$ th diffraction order $d_{m}$. With this setup it should be possible to determine 550 fluorescence spectra with a spectral resolution of 2 to $3 \mathrm{~nm}$ in a range from 360 to $510 \mathrm{~nm}$.

\subsection{Two-dimensional spectral camera}

To avoid mechanical scanning of cartilage samples with the one-dimensional system described above, another approach based on CTIS was developed for fast determination of fluorescence spectra for every point of a two-dimensional sample. CTIS was invented separately by Okamoto, Yamaguchi, Bulygin and Vishnyakov (Okamoto and Yamaguchi, 1991; Bulygin and Vishnyakov, 1992). The setup presented in Fig. 1 is very similar to the setup of the one-dimensional system. The first lens which images the sample in the aperture plane is replaced by another fast lens (Ricoh, FL-CC2514$5 \mathrm{M})$ with a higher transmittance in the spectral region around $400 \mathrm{~nm}$. The former slit aperture is replaced by a square one of about $127 \mu \mathrm{m} \times 127 \mu \mathrm{m}$ made of four razor blades (Wilkinson Sword Classic) taped on a mounting plate. Thus, the aperture can be easily replaced and aligned. The $35 \mathrm{~mm}$ lens (Edmund Optics, Techspec, \#59-872) is employed as a collimating lens here. To obtain the two-dimensional diffraction pattern needed for CTIS, the blazed line grating is replaced by a cross-grating of 200 lines $\mathrm{mm}^{-1}$ (Astromedia, Multispektralfolie). The re-imaging lens applied in this setup is a fast $16 \mathrm{~mm}$ lens with higher transmittance in the spectral region around $400 \mathrm{~nm}$ (Ricoh, FL-CC1614-5M). The numerical aperture of the complete lens arrangement is about 0.25 .

The observed two-dimensional diffraction pattern can be considered as parallel projections of voxels of a threedimensional object cube. Two dimensions of this threedimensional cube are the spatial coordinates and one dimension is the wavelength (Okamoto and Yamaguchi, 1991; Bu- lygin and Vishnyakov, 1992). The voxels of the object cube can be denoted as a long serialized vector $f$. The effect of the imaging system can be described by a matrix $\mathbf{H}$ with the dimension of $M \times N$, where $N$ is the number of voxels in the object cube and $M$ is the number of pixels in the image. For every point of the aperture a matrix $\mathbf{H}$ is created with the use of Eq. (1). Operating the system matrix $\mathbf{H}$ on the object cube vector $\boldsymbol{f}$ gives vector $\boldsymbol{g}$ as described in Eq. (2),

$g=\mathbf{H} f$

representing the serialized pixels in the image. Based on the projections, the three-dimensional object cube can be reconstructed (Descour and Dereniak, 1995).

A common and appropriate algorithm is the expectation maximization algorithm in Eq. (3) (Dempster, 1977):

$\hat{\boldsymbol{f}}_{n}^{k+1}=\frac{\hat{\boldsymbol{f}}_{n}^{k}}{\sum_{m=1}^{M} \mathbf{H}_{m n}} \sum_{m=1}^{M} \mathbf{H}_{m n}^{T} \frac{\boldsymbol{g}_{m}}{\mathbf{H} \hat{\boldsymbol{f}}^{k}}$.

As a starting point the pixel intensity of the recorded image was used as first guess for $\boldsymbol{f}^{0}=\mathbf{H}^{\mathrm{T}} \boldsymbol{g} . \boldsymbol{f}$ converges sufficiently after 10 iterations. All calculations are performed with MATLAB ${ }^{\circledR}$ R2013a on a Fujitsu personal computer with 8 GB RAM and an Intel ${ }^{\circledR}$ core i5 processor.

\subsection{Cartilage samples}

Because of the fact that the cartilage composition is very complex, at the beginning only samples with assumed pure collagen I and collagen II are examined. The examined cartilage specimens are small round slices with a size of about $0.25 \mathrm{~cm}^{2}$. Three slices of collagen I which are extracted from porcine meniscus and three slices of collagen II which were taken from porcine septum are investigated. These samples were kindly provided by the Ulm University Medical Center.

\section{Results}

\subsection{Result of the one-dimensional spectral camera setup}

Figure 2 shows the observed diffraction pattern for collagen I and II fluorescence at $365 \mathrm{~nm}$ excitation, and in Fig. 3 the corresponding calculated spectra of collagen I and II for a single point of the line sample are presented.

The fluorescence maximum for collagen I is around $435 \mathrm{~nm}$, and for collagen II it can be found around $428 \mathrm{~nm}$. The fluorescence intensity of collagen II is much lower than the fluorescence intensity of collagen I. The observed maximum at $375 \mathrm{~nm}$ is no fluorescence but reflected and scattered LED emission. The observed spectral shift and the different fluorescence intensities allow a future distinguishing between collagen I and II. Figure 4 presents a complete fluorescence data set of a collagen I sample along a line with 


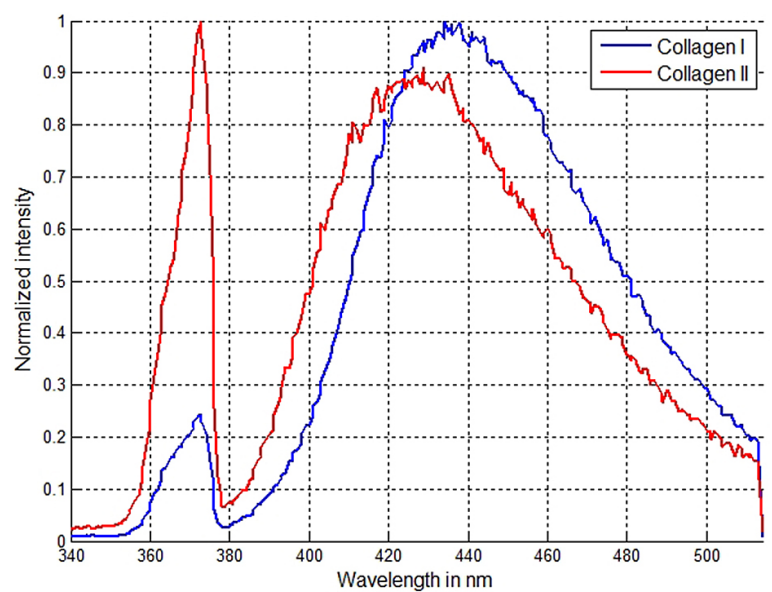

Figure 3. Calculated single fluorescence spectra of collagen I and II with $365 \mathrm{~nm}$ excitation wavelength. Integration time: $10 \mathrm{~s}$ for collagen I, $60 \mathrm{~s}$ for collagen II.

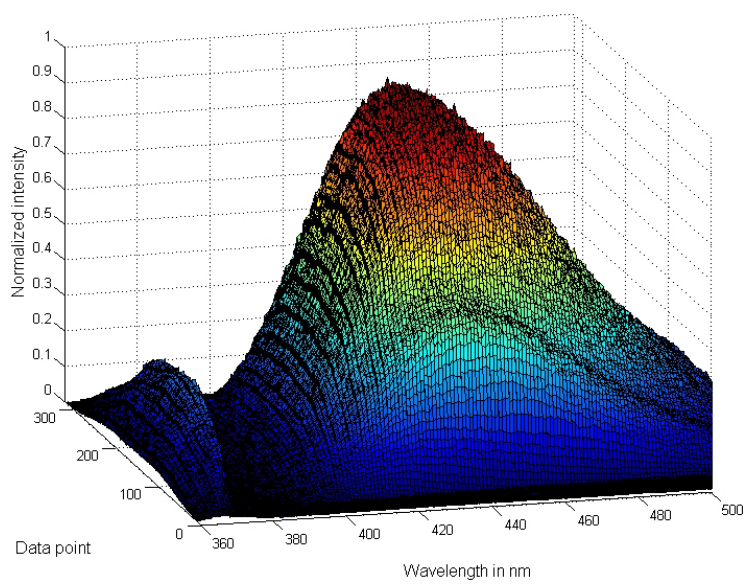

Figure 4. Several hundred simultaneously recorded fluorescence spectra of collagen I with $365 \mathrm{~nm}$ excitation. Integration time: $10 \mathrm{~s}$.

more than 300 fluorescence spectra. The spectrum of collagen I in Fig. 3 is just one data point of the data set shown in Fig. 4. The same trend can be found for an excitation wavelength of $340 \mathrm{~nm}$.

\subsection{Result of the two-dimensional spectral camera setup}

To check the functionality of the two-dimensional system, as a first test the spectrum of an energy-saving lamp was determined as described above and compared to the results of a commercial spectrometer (Avantes, SensLine AvaSpec-2048 $\mathrm{XL}$ ). Figure 5 shows the recorded diffraction pattern, and in Fig. 6 the comparison between the calculated spectrum and the spectrum measured with the spectrometer can be found.

For wavelengths between 425 to $650 \mathrm{~nm}$ the results of the spectral camera and the commercial spectrometer are very similar. The peak emissions can be found at the same wave-

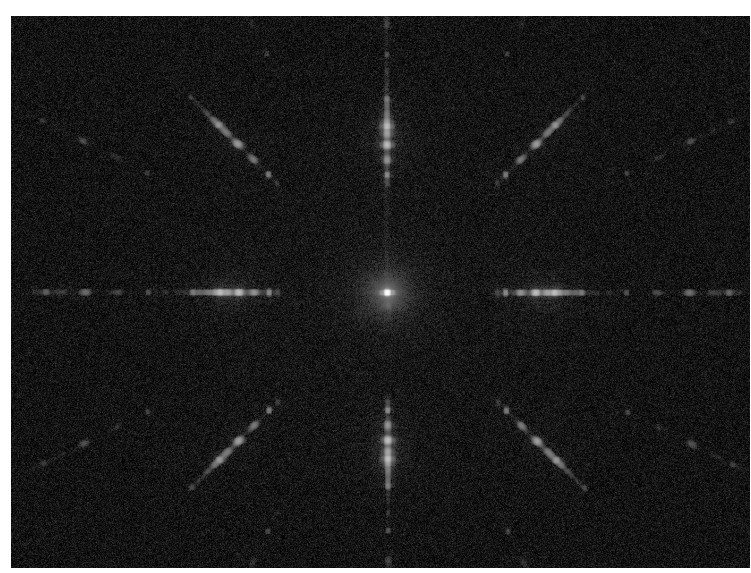

Figure 5. Diffraction pattern of the energy-saving lamp.

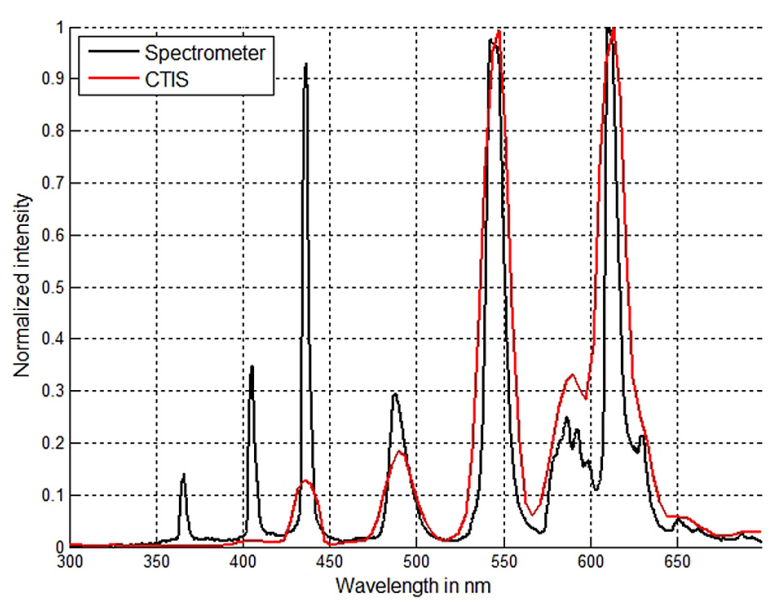

Figure 6. Spectrum of an energy-saving lamp acquired with the two-dimensional spectral camera (red) and a spectrometer (black).

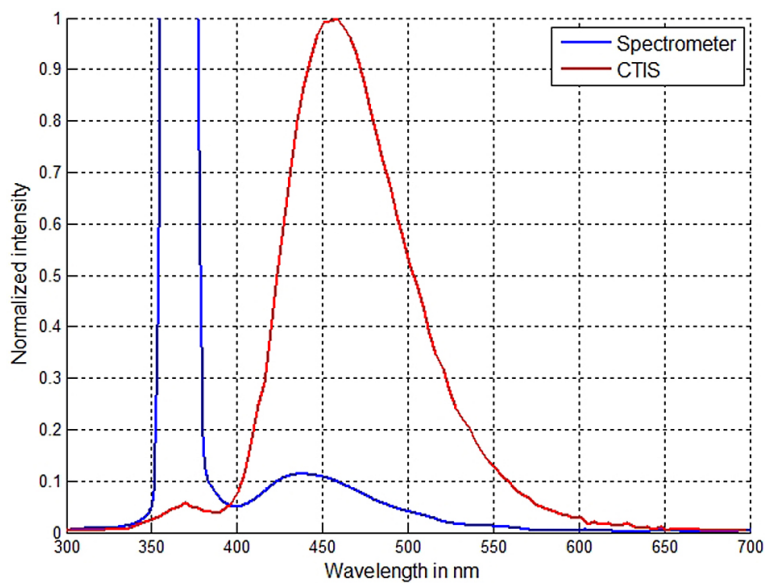

Figure 7. Single-point fluorescence spectrum of collagen I after $365 \mathrm{~nm}$ excitation, acquired with the two-dimensional spectral camera (red) and the spectrometer (blue). 


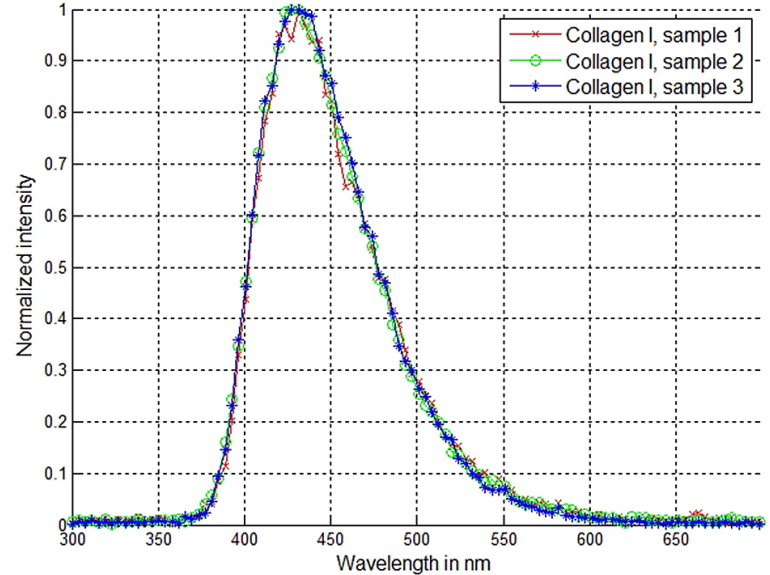

Figure 8. Fluorescence spectra of collagen I (meniscus) with $340 \mathrm{~nm}$ excitation. The exposure time is $1200 \mathrm{~s}$.

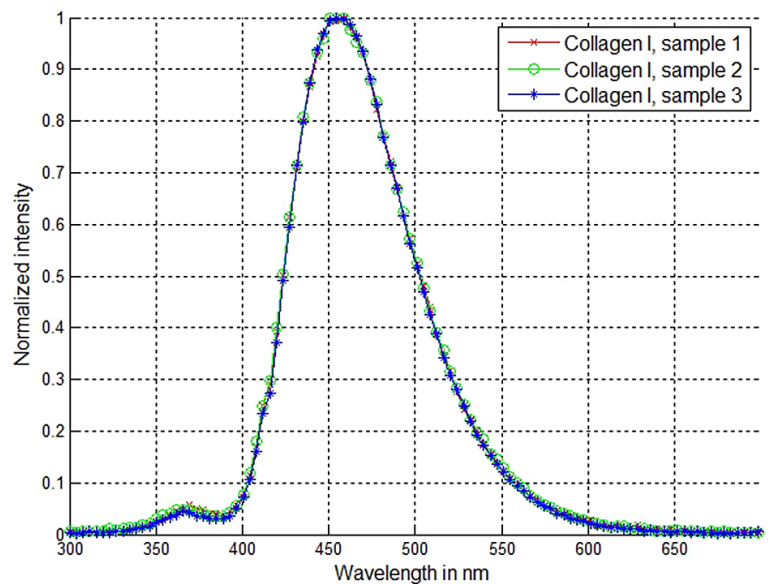

Figure 9. Fluorescence spectra of collagen I (meniscus) with $365 \mathrm{~nm}$ excitation. The exposure time is $0.3 \mathrm{~s}$.

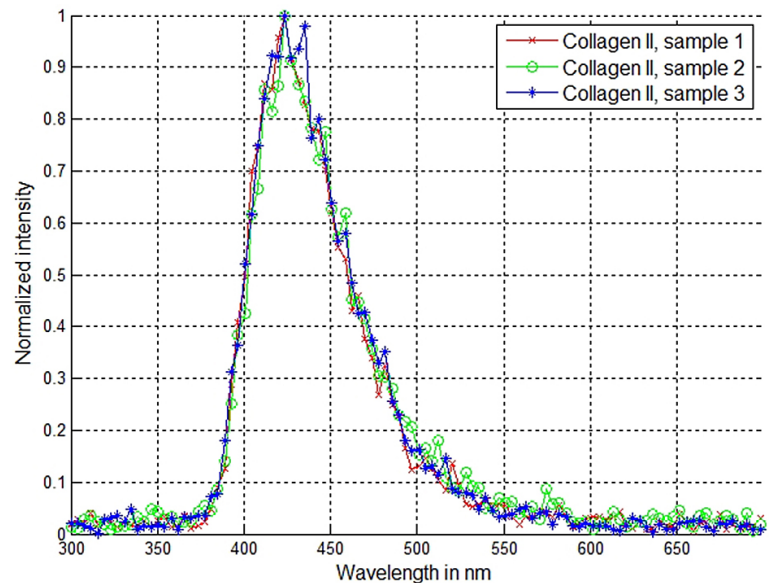

Figure 10. Fluorescence spectra of collagen II (septum) with $340 \mathrm{~nm}$ excitation. The exposure time is $1200 \mathrm{~s}$.

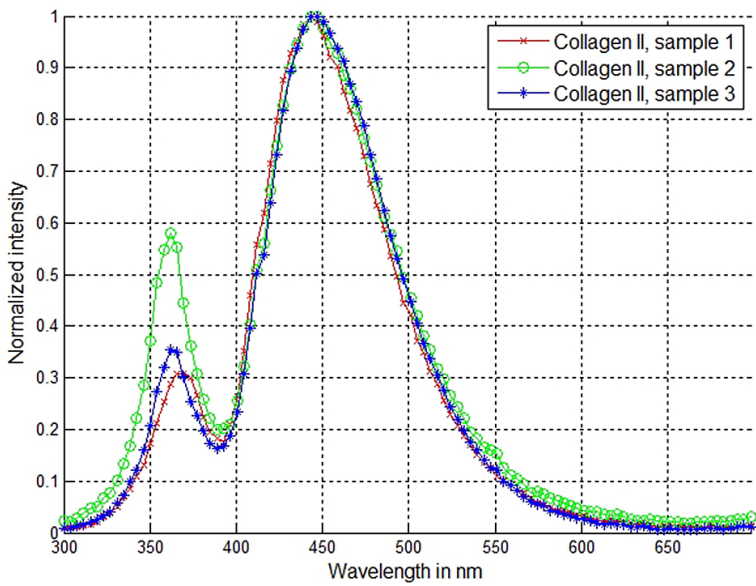

Figure 11. Fluorescence spectra of collagen II (septum) with $365 \mathrm{~nm}$ excitation. The exposure time is $0.3 \mathrm{~s}$.

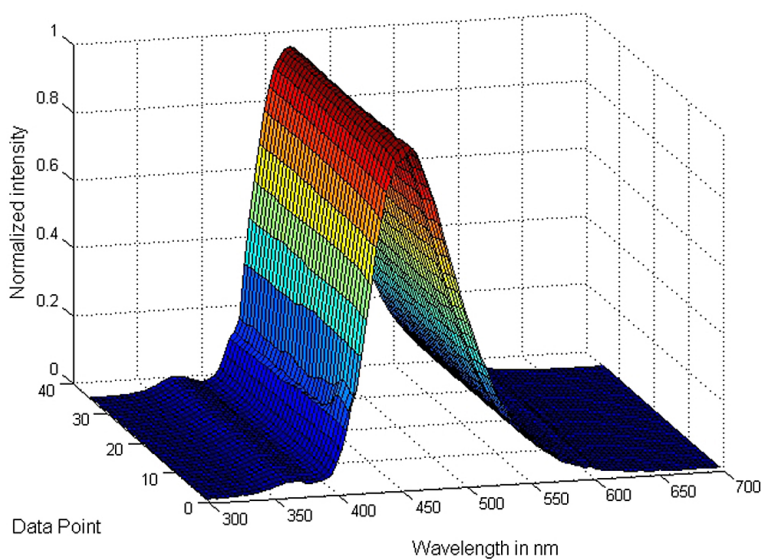

Figure 12. Collagen I fluorescence spectra of $7 \times 5$ points arranged in a line for presentability; the excitation wavelength is $365 \mathrm{~nm}$.

length; however the peaks recorded with the spectral camera are broader, which is caused by a lower spectral resolution of about $10 \mathrm{~nm}$. Another observable feature is the lower intensity of the spectral camera below $450 \mathrm{~nm}$, which can be attributed to the transmission properties of the three lenses in the optical system, even though they have been selected because of a higher transmission compared to most commercial lenses.

A comparison between the determined fluorescence spectra of collagen I with the commercial spectrometer and the spectral camera is depicted in Fig. 7. The fluorescence maximum of collagen I measured with the spectrometer is around $440 \mathrm{~nm}$ and matches the results of the one-dimensional camera system in Fig. 3 very well. The two-dimensional camera system shows a peak shift to $455 \mathrm{~nm}$. This is probably due the lower resolution and the sensitivity dependence on the wavelength caused by the lenses that was already observed in Fig. 6. 
Despite this spectral shift it is still possible to distinguish collagen I and collagen II with the two-dimensional system because both fluorescence spectra are shifted. Measured and calculated fluorescence spectra for single points of the collagen samples for collagen I and II and 340 and $365 \mathrm{~nm}$ excitation are depicted in Figs. 8 to 11.

For the $365 \mathrm{~nm}$ excitation 10 images with an exposure time of $0.3 \mathrm{~s}$ were taken and averaged to smooth the spectrum. For the low-intensity $340 \mathrm{~nm}$ LED the exposure time was set to $1200 \mathrm{~s}$ and only a single picture was recorded.

The results of the different collagen samples show that the determination of the spectra is very reproducible.

At an excitation wavelength of $340 \mathrm{~nm}$ the fluorescence maximum of collagen I is around $430 \mathrm{~nm}$ and of collagen II around $423 \mathrm{~nm}$. A greater difference between both emission peaks is observable with an excitation wavelength of $365 \mathrm{~nm}$. Here, the fluorescence peak emission can be found around $455 \mathrm{~nm}$ for collagen I and $443 \mathrm{~nm}$ for collagen II. The smaller maxima in Figs. 9 and 11 are caused by reflected light from the LED. Moreover, the reflections of the collagen II samples are pronounced differently in terms of their intensity (see Fig. 11).

Figure 12 shows $7 \times 5$ spectra of a collagen I sample excited with $365 \mathrm{~nm}$. The calculation of the spectrum with the expectation maximization algorithm takes 4 to $7 \mathrm{~s}$ for each sample point.

\section{Conclusions}

Two spectral camera systems for cartilage fluorescence detection in the spectral range from 380 to $500 \mathrm{~nm}$ are developed and evaluated. A Peltier cooled monochromatic camera and fast lenses allow the detection of weak fluorescence signals. The one-dimensional system offers a high sensitivity and a high spatial resolution despite a simple setup and an elementary algorithm for the calculation of the spectra. The most important cartilage compounds collagen I and II can be determined and discriminated. The only drawback is the need for a mechanical scanning device if not only a line but a two-dimensional sample has to be examined.

With small changes in the setup, the spectral camera system can even be extended to direct two-dimensional spectra determination on cartilage samples using the CTIS approach. For 340 and $365 \mathrm{~nm}$ and for collagen I and collagen II, fluorescence spectra can be determined. The spectra are very reproducible, and collagen I and II seem to be easily distinguishable. The current two-dimensional system is able to deliver $9 \times 9$ spectra with a single snap shot and a resolution of about $10 \mathrm{~nm}$.

The future goal is to determine the spectra of $100 \times 100$ points in reasonable time. Therefore, the aperture probably has to be enlarged, which in turn leads to a reduction of spatial resolution. One approach is the optimization of the algorithm; another one is the improvement of the optical system. After optimization of the system other important fluorescing cartilage components (e.g. elastin, GAG) will be included in the cartilage analysis.

Acknowledgements. Support from the Bundesministerium für Bildung und Forschung (Förderkennzeichen 03FH008I3), the Ulm University Medical Center (N. Rotter and S. Schwarz of the Department of Oto-Rhino-Laryngology Head and Neck Surgery) and the Institut für Lasertechnologien in der Medizin und Messtechnik Ulm (C. Scalfi-Happ and K. Stock) is gratefully acknowledged.

Edited by: A. König

Reviewed by: two anonymous referees

\section{References}

Bulygin, T. V. and Vishnyakov, G. N.: Spectrotomography - a new method of obtaining spectrograms of two-dimensional objects, Analytical Methods for Optical Tomography, P. Soc. Photo-Opt. Ins., 1843, 315-322, 1992.

Dempster, A. P., Laird, N. M., and Rubin, D. B.: Maximum Likelihood from Incomplete Data via the EM Algorithm, J. Roy. Stat. Soc. B. Met., 39, 1-38, 1977.

Descour, M. R. and Dereniak, E. L.: Nonscanning no-moving-parts imaging spectrometer, P. Soc. Photo-Opt. Ins. 2480, 48-64, 1995.

Habel, R., Kudenov, M., and Wimmer, M.: Practical Spectral Photography, Comput. Graph. Forum, 31, 449-458, 2012.

Kirkpatrick, N. D., Hoying, J. B., Botting, S. K., Weiss, J. A., and Utzinger, U.: In vitro model for endogenous optical signatures of collagen, J. Biomed. Opt., 11, 054021, 2006.

Kuehn, A., Graf, A., Wenzel, U., Princz, S., Miller, R., Mantz, H., and Hessling, M.: Development of a spectral camera for cartilage monitoring, AMA Conference, Nuremberg, Germany, May 2015, 807-811 2015.

Langer, R. and Vacanti, J. P.: Tissue engineering, Science, 260, 920926, 1993.

Lefebvre, V., Peeters-Joris, C., and Vaes, G.: Production of collagens, collagenase and collagenase inhibitor during the dedifferentiation of articular chondrocytes by serial subcultures, BBAMol. Cell Res., 1051, 266-275, 1990.

Okamoto, T. and Yamaguchi, I.: Simultaneous acquisition of spectral image information, Opt. Lett., 16, 1277-1279, 1991.

Sittinger, M., Bujia, J., Rotter, N., Reitzel, D., Minuth, W. W., and Burmester, G. R.: Tissue engineering and autologous transplant formation: Practical approaches with resorbable biomaterials and new cell culture techniques, Biomaterials, 17, 237-242, 1996.

Skalak, R. and Fox, C. F.: Tissue engineering: Proceedings of a workshop held at Granlibakken, Lake Tahoe, CA, USA, 1988.

Tuan, V.-D. and Brian, M. C.: Fluorescence spectroscopy for biomedical diagnostics, in: Biomedical Photonics Handbook, edited by: Vo-Dinh, T., Florida: CRC Press, Boca Raton, USA, 1-51, 2003.

Uchiyama, A., Inoue, T., and Fujimoto, D.: Synthesis of pyridinoline during in vitro aging of bone collagen, J. Biochem., 90, 1795-1798, 1981.

Von der Mark, K., Gauss, V., Von der Mark, H., and Müller, P.: Relationship between cell shape and type of collagen synthesised as chondrocytes lose their cartilage phenotype in culture, Nature, 267, 531-532, 1977. 